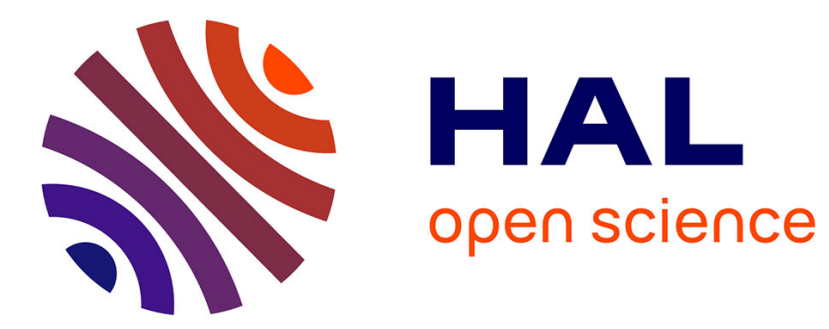

\title{
L'acte éditorial : vers une théorie du geste Brigitte Ouvry-Vial
}

\section{To cite this version:}

Brigitte Ouvry-Vial. L'acte éditorial: vers une théorie du geste. Communication \& langages, 2007, 154, pp.59-74. 10.3406/colan.2007.4691 . hal-01812157

\section{HAL Id: hal-01812157 https://hal.science/hal-01812157}

Submitted on 11 Jun 2018

HAL is a multi-disciplinary open access archive for the deposit and dissemination of scientific research documents, whether they are published or not. The documents may come from teaching and research institutions in France or abroad, or from public or private research centers.
L'archive ouverte pluridisciplinaire HAL, est destinée au dépôt et à la diffusion de documents scientifiques de niveau recherche, publiés ou non, émanant des établissements d'enseignement et de recherche français ou étrangers, des laboratoires publics ou privés. 


\section{L'acte éditorial :} vers une théorie du geste

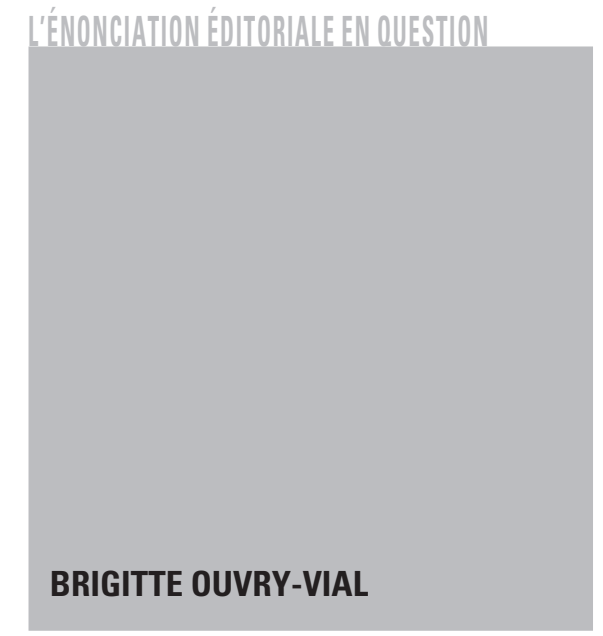

Longtemps occulté de la conscience des lecteurs eux-mêmes, l'acte éditorial est devenu progressivement un objet d'études critiques, nouveau tant par l'intérêt pluridisciplinaire qu'il suscite que par la nécessité même de l'approche interdisciplinaire qu'il implique. Partant des travaux existants et des enjeux de ce sujet émergeant, au croisement de la théorie littéraire, des sciences de la communication et de l'histoire culturelle, cet article traite des approches à l'œuvre dans l'analyse de la médiation éditoriale qui pourrait trouver dans le champ de la médiologie des éléments théoriques adaptés à la diversité à la fois historique et contextuelle de ses pratiques. II considère en particulier la question de la réception éditoriale et propose d'englober les concepts d'écriture et d'énonciation éditoriale dans la notion plus large de geste, soit une gestualité éditoriale antérieure à la production d'une représentation signifiante.

1. C'est le champ disciplinaire du Book History.

2. Telle que définie par Régis Debray, en particulier dans Introduction à la médiologie, PUF, 2000.

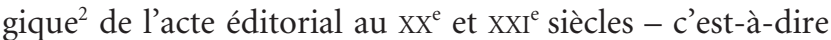
l'étude des pratiques culturelles et intellectuelles des éditeurs contemporains comme des formes symboliques ui en découlent -, n'existe pas en tant que telle:

Les éléments disponibles pour la reconstituer ne sont pas regroupés autour de revues, de groupes de recherches institués et visibles mais dispersés dans une

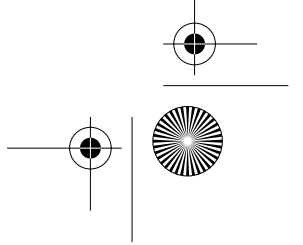


diversité d'études, travaux ou documents où, d'une part, on ne songe pas forcément à aller les chercher et dont, d'autre part, ils ne constituent pas l'objectif premier en terme d'information scientifique.

- La nature, les conditions concrètes et l'impact communicationnels de la médiation éditoriale, en littérature générale, au $\mathrm{XX}^{\mathrm{e}}$ et $\mathrm{XXI}^{\mathrm{e}}$ siècle, ne font pas l'objet d'une approche théorique suffisante ou proportionnelle à l'importance quantitative, sociologique et médiatique de la production éditoriale elle-même dans ce domaine, quelle que soit l'ère géographique ou linguistique considérée.

- Les études sur le livre et l'édition impliquent une réflexion sur la langue et ses usages, sur les types et les situations de discours écrits, sur les techniques de rédaction que ces situations appellent et intéressent un public de recherche pluridisciplinaire (venant autant des LSH, des SIC, des études de documentation, de la bibliothéconomie) mais supposent aussi des praticiens ayant une double compétence, universitaire et professionnelle, de la médiation éditoriale.

- Il manque une étude d'ensemble portant sur la conception de la médiation éditoriale telle qu'elle se traduit dans le livre, à travers une conjonction pensée, délibérée, signifiante, d'éléments matériels (mise en page, format, typographie, couverture, papier...) et d'éléments textuels (notes, avertissement, remerciements, préface, achevé d'imprimer, quatrième de couverture...).

Bien entendu, on doit à Gérard Genette une étape notable de cette étude d'ensemble qui, partant d'une vue d'ensemble du livre lui-même et d'une prise en compte de la diversité matérielle des éléments indicateurs de son processus de réalisation, propose dans Seuils une typologie et une définition du paratexte dans sa complexité "d'objet [...] multiforme et tentaculaire » ${ }^{3}$. Mais, comme Genette lui-même le constate en conclusion, cette vue d'ensemble synthétique, au-delà du parcours très élaboré qu'il en propose, relève d'une vue de l'esprit et «n'atteint nullement à une exhaustivité à laquelle il ne prétendait d'ailleurs pas $»^{4}$. Non seulement les différents éléments paratextuels identifiés et évoqués demandent chacun à soi seul une enquête approfondie, mais encore Genette attire-t-il l'attention en conclusion - au titre des lacunes provisoires de son traité -, sur «trois pratiques dont la pertinence paratextuelle [me] paraît indéniable ${ }^{5}$ et non des moindres, la traduction, la publication en feuilleton, l'illustration. De plus, les analyses du paratexte ou de l'épitexte éditorial portent sur des cas où la responsabilité et les choix émanent d'abord de l'auteur, et supposent que le discours éditorial en général, subordonné « aux nécessités du commerce » et aux usages établis, est essentiellement consensuel et impersonnel.

Si l'étude du livre et de l'édition s'est enrichie depuis une dizaine d'années de nombreuses analyses s'attachant à des éditions d'ouvrages précis, à des monogra-

3. Gérard Genette, Seuils, Le Seuil, 1987, p. 374.

4. Ibid., p. 372.

5. Ibid.

6. Ibid., p. 318.

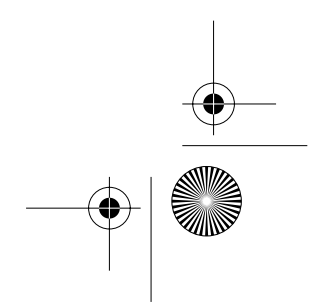


phies d'éditeurs, aux évolutions historiques et contextuelles des métiers, arts, pratiques du livre ou de la lecture, un traité unique, synthétique et au demeurant complet du paratexte éditorial paraît donc impossible. Toutefois, «l'exhortation » formulée par Genette ${ }^{7}$ souligne la nécessité et l'importance de fédérer les études du paratexte comme l'inventaire de ses formes et modalités, et en particulier à partir de l'intention, ou intentionnalité, dont ce paratexte pourrait diversement relever. Constatant en conclusion du chapitre final de Seuils que «la boucle est bouclée: partie de l'édition, notre enquête revient à l'édition. L'ultime destin du paratexte est de tôt ou tard rejoindre son texte, pour faire un livre ${ }^{8}$, Genette suggère implicitement l'intérêt d'une réflexion sur les manières d'éditer qui sont des manières, diverses et propres à des personnes en particulier, plus qu'à une fonction générale d'édition, de lire, de dire, de restituer, accompagnée d'une description in situ, expérimentale, clinique, de l'atelier, des outils et des effets de l'acte éditorial.

\section{INTENTIONNALITÉ, MEDIUM}

On ne reviendra pas ici sur les fonctions de l'éditeur, ni sur la fonctionnalité du paratexte, pas plus qu'on ne rentrera dans un détail des intentions possibles des éditeurs, qu'elles se présentent comme proprement littéraires, confidentielles, plus largement communicationnelles ou encore exclusivement commerciales. En deçà même de ces analyses, force est de constater qu'on ignore, sur le fond, les objectifs des éditeurs vis-à-vis des textes et des lecteurs? S'agit-il d'établir les conditions d'une lecture immédiate, facile, par un lecteur ordinaire, ce qui ne suppose pas nécessairement la préparation d'un texte au rabais? S'agit-il de provoquer une émotion esthétique? Ne pourrait-il s'agir, avant tout, de travailler la performance ${ }^{9}$ du texte pour le rendre accessible à un lecteur compétent ? Ou de l'adapter tout simplement à un public socio-historique donné, fût-il incompétent ? Si on suppose que le respect du texte et des intentions d'auteur est la base du processus d'édition, on sait bien aussi que ce respect dépend de la perception et interprétation éditoriales qui sont faites aussi bien des textes, des desseins de l'auteur que de la capacité des lecteurs à se les représenter, sans oublier le principe désormais acquis et justement démontré d'une liberté active de chaque lecture au cours de laquelle il "arrive quelque chose ${ }^{10}$ au livre. Moins que d'intentions éditoriales spécifiques, que les études de cas décryptent, on parlera globalement d'une intentionnalité éditoriale, de nature critique ${ }^{11}$, qui ne se résume pas au

7. Ibid., p. 371.

8. «L'épitexte privé », ibid., p. 370.

9. Pour cette notion, on renverra à notre article «Performance du texte dans le livre ou le rôle de l'éditeur dans son accomplissement", in Textes en Performances, Actes du colloque Cernet, novembre 2003, Ambroise Barras et Éric Eigenmann [éds], MétisPresses, Genève, 2006, pp. 171-182.

10. L'expression est de Borges, rapportée in Richard Burgin, Conversations avec J.-L. Borges, Paris, Gallimard, 1972. Sur «l'avènement» du texte dans la lecture éditoriale, cf. B. Ouvry-Vial, «Performance du texte dans le livre ou le rôle de l'éditeur dans son accomplissement ", in Textes en Performances, op. cit., pp. 174-175.

11. Cf. B. Ouvry-Vial, « Jean Paulhan et Gaëtan Picon : des éditeurs entre tradition et transmission », in L'Acte éditorial. Publier à la Renaissance et aujourd'hui, Actes du colloque réunis par Brigitte OuvryVial et Anne Réach Ngô, Textuels, Revue de l'UFR LAC/Université Paris7-Denis Diderot, janvier 2008. 
paratexte mais y est perceptible, repérable, qui lui confere son caractère " transitif ", «d'instrument d'adaptation $»^{12}$ aux modifications du public et plus largement son action «de l'ordre de l'influence, voire de la manipulation $»^{13}:$ au-delà du paratexte, qui peut émaner des auteurs comme des éditeurs, exclusivement ou conjointement les uns aux autres, il faut supposer une intentionnalité présidant aux opérations formelles d'édition et qui consiste à manipuler, s'approprier, user d'éléments de compréhension du texte et du livre provenant de cultures, de genres ou encore d'époques différentes; à les rendre lisibles ou visibles dans le livre par une sorte "d'encodage " destiné à adapter le texte aux pratiques de lecture $^{14}$; à aménager, faciliter ou orienter l'accès des lecteurs au texte (c'est le cas des variations de formats, de collections, des passages de la $1^{\text {re }}$ édition à une réédition en poche...); à restituer aussi une dimension du texte perdue ou occultée par la succession des éditions dans le temps (c'est le cas des éditions critiques, des nouvelles éditions, des traductions revues et corrigées). Le résultat visé ou le but atteint par les opérations éditoriales - dont la liste évoquée ci-dessus n'est bien entendu pas exhaustive - étant au minimum un éclairage du texte, au maximum la création d'un sens tiers.

Une analyse globale (communicationnelle et littéraire, appuyée sur des corpus caractérisés dans leur contexte historique) devrait dès lors permettre d'observer et d'étudier le processus éditorial de transmission des textes et des idées, conjointement - et non de façon distincte - à l'invention des formes et modalités de cette transmission. Elle prendrait ainsi en compte le double caractère du livre comme véhicule de transmission, comme medium, tel que le décrit Régis Debray : «Certaines de ses propriétés s'ordonnent à un pôle matériel (le papier, l'imprimerie, la forme), et d'autres à un pôle social (langue, nation, éditeur). D’un côté, une matière organisée ; de l'autre, des organisations matérialisées ${ }^{15}$.»

La réflexion critique sur l'activité éditoriale ne vise pas, on l'aura compris, une sociologie de la lecture à proprement parler, mais la mise en évidence des liens intimes entre écrire, lire et éditer qui font de l'édition une réponse aux pratiques contemporaines de lecture et aux besoins dont elles témoignent. Considérer le livre comme medium et l'analyser en tant que tel, consisterait non pas tant à en décrire la façon, à reconstituer l'historique de sa fabrication qu'à étudier conjointement les choix matériels et intellectuels, techniques et esthétiques mis en œuvre pour la transmission du texte, pour sa mise en livre et à étudier les raisons, les moyens, les effets de ces choix sur la lecture.

Un ouvrage critique récemment paru aux États-Unis, $M a(r)$ king of the Text, sous-titré "The presentation of meaning on the literary page ${ }^{16}$ relève et témoigne, par son titre déjà, de cette démarche méthodologique et de cette

12. G. Genette, Seuils, op. cit., p. 375.

13. Ibid., p. 376.

14. C'est le propos de Roger Chartier dans son article «Du livre au lire», in R. Chartier, Pratiques de la lecture, Marseille, Rivages, 1985, pp. 62-88.

15. Régis Debray, Introduction à la médiologie, op. cit., p. 39.

16. Ma(r) king the Text, Edited by Joe Bray, Miriam Handley and Anne C. Henry, Ashgate, 2000.

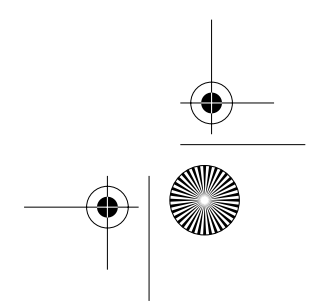


conception théorique de l'acte éditorial selon lequel «to mark a text is to make it $\aleph^{17}$. Une conception partagée par éditeurs et auteurs, même si une bonne part des articles rassemblés dans ce volume collectif, et qui s'intéressent à " this fringe between text and paratext, questioning the terms of this opposition ${ }^{18}$, font plus état du travail d'édition des auteurs que de leurs éditeurs. L'article "To correspond with you in Green-Covered Volumes ${ }^{19}$, consacré à la production éditoriale des livres de George Eliot et à l'attention que l'auteur et son mari G.E. Lewes apportèrent tant à la mise en livre du texte qu'à l'apparence physique du livre considérée comme faisant partie du travail créateur d'écriture, détaille la conception des liens entre la physique du livre et la perception et réception du texte par le lecteur: plus qu'une simple stratégie économique, la conception éditoriale des ouvrages de G. Eliot fonctionne "both as an art form and as a set of assumptions by reflecting as well as influencing the culture of middle-class Victorian readers ${ }^{20} . \mathrm{Si}$ les choix formels - format, visuel et couleur de la couverture, publication en feuilleton... - incarnent les valeurs culturelles et sociales des lecteurs de G. Eliot et répondent à leurs attentes, ils contribuent aussi à modeler et orienter autant le processus d'écriture que de lecture, conformément au souci de l'auteur de «correspond with you $»^{21}$, d'adapter, correspondre, dialoguer avec le lecteur à travers et par un livre, conçu comme carte d'identité du texte.

Si le livre en effet est le medium attitré de l'œuvre littéraire, tous les livres ne sont pas, n'ont pas automatiquement valeur de medium, terme qui désigne "plusieurs réalités de nature différente [qui] se superposent souvent mais ne peuvent en aucun cas se confondre $[\ldots]: 1 /$ Un procédé général de symbolisation ; 2 / un code social de communication ; 3 / un support physique d'inscription et de stockage et $4 /$ un dispositif de diffusion avec le mode de circulation correspondant $»^{22}$. L'éventuelle superposition de ces strates dans le livre, ou le fait qu'ils relèvent plus ou moins effectivement de ces divers ordres ne suffit pas à faire de tout livre un medium en soi. Il faut encore qu'on y retrouve le double corps du medium, répondant aux deux versants combinés du processus de transmission ${ }^{23}$. Le processus de transmission peut être initié et étroitement contrôlé par l'auteur : c'est le cas dans de nombreuses éditions du XIX', comme en attestent, entre autres exemples, la correspondance de Mallarmé avec ses éditeurs Lemerre, Dujardin, Vanier... ${ }^{24}$, celle ou de Baudelaire avec Poulet-

17. Ibid., p. xvii. Traduction : «marquer un texte c'est le faire/fabriquer ».

18. Ibid., p. xix. Traduction : «cette limite entre texte et paratexte, en interrogeant la nature de cette opposition ".

19. Ibid., pp. 12-25. Traduction : "Correspondre avec vous/ vous correspondre dans des ouvrages à couverture verte ».

20. Traduction : " À la fois comme une forme artistique et un ensemble de suppositions reflétant et influençant la culture des lecteurs des classes moyennes de l'époque victorienne. Linda K. Hughes et Michael Lund, cités par Gail Elizabeth Korn, " To correspond with You in Green-Covered Volumes ", $\mathrm{Ma}(\mathrm{r})$ king the Text, op. cit., p. 15.

21. Voir note 19.

22. Régis Debray, Introduction à la médiologie, op. cit., p. 35.

23. Ibid., p. 39.

24. Stéphane Mallarmé, Correspondance, recueillie, classée et annotée par Henri Mondor [et Lloyd James Austin], Tome II et III, Gallimard, 1959-1985.
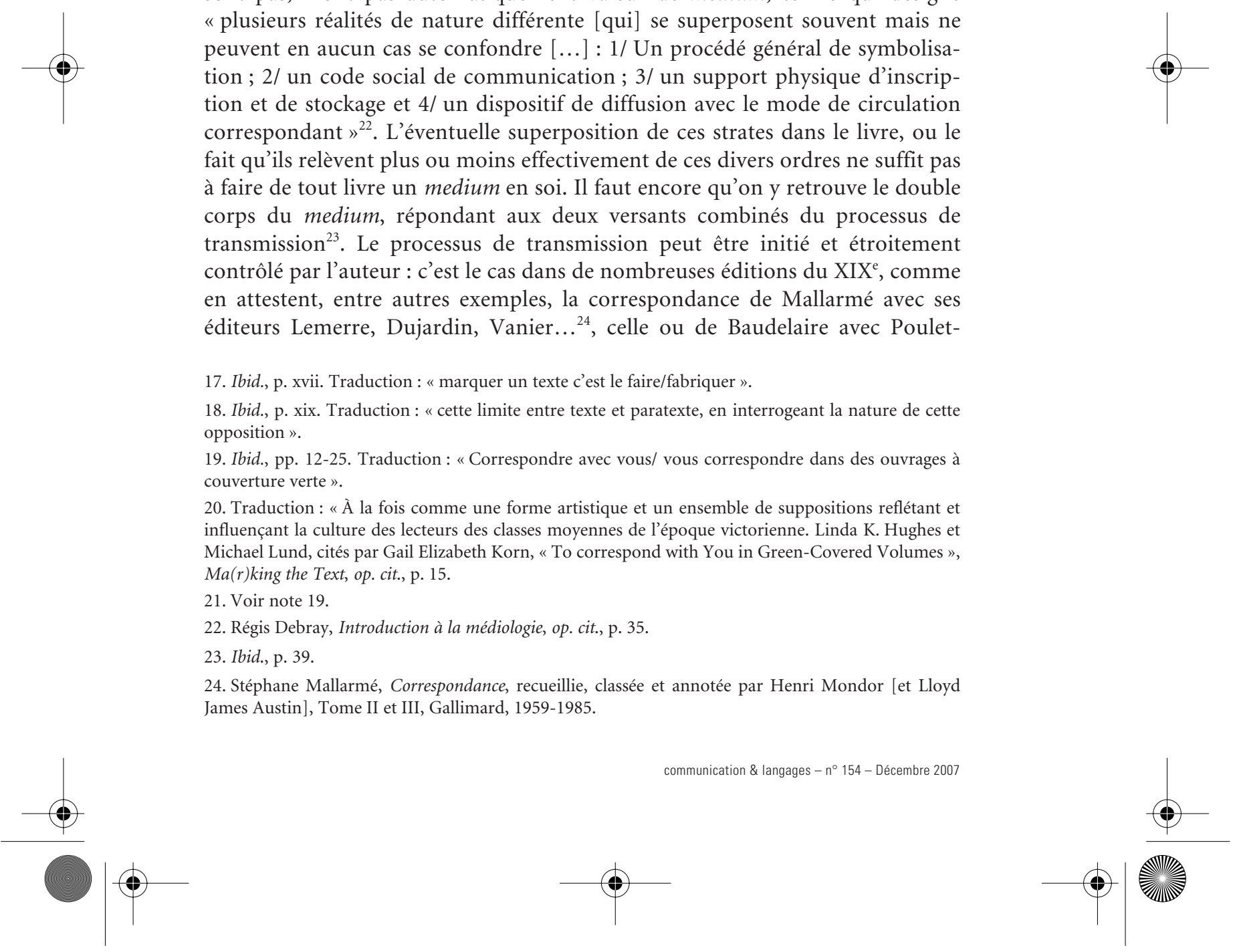
Malassis $^{25}$. Le processus de transmission peut aussi être contrôlé par l'éditeur : c'est principalement le cas au XXe, en témoignent correspondances, documents d'archives, traités sur des éditeurs à forte notoriété comme Gallimard, plus confidentiels comme Pierre-André Benoît ou Bruno Roy, ou encore sur l'art de faiseurs de livres comme Massin ${ }^{26}$ et Faucheux ${ }^{27}$. Le geste peut être personnel ou collectif, peu importe. Ce qui compte c'est la combinaison de la techne (action de l'homme sur les choses) et de la praxis (action de l'homme sur l'homme $)^{28}$, de «l'organisation de la matière inorganique et de l'organisation $\mathrm{du}$ socius ${ }^{29}$; c'est la conception préalable du livre non comme un objet, une chose mais comme "une place et une fonction dans un dispositif véhiculaire $»^{30}$, c'est « la mise en fonction médium de l'objet $\mathrm{x}$ [ici, le livre] qui ne l'est pas par lui-même $»^{31}$.

Outre que cette attention au "parcours - du conditionné au conditionnant", «aux signes», "au regard [plus qu'] au message ou à l'image » ${ }^{32}$ caractérise l'approche médiologique, on voit bien ce que la considération de l'intention qui préside au livre pourrait apporter au questionnement récurrent sur la valeur respective des livres, sur la différence entre livre et livre, sur l'originalité ou la vulgarité littéraire, questionnement qui émane autant des auteurs, des éditeurs, des lecteurs que des critiques ${ }^{33}$.

\section{Peut-on parler de Réception éditoriale?}

Quel que soit le jugement positif ou négatif qu'on porte sur lui, c'est bien l'aspect médiateur du travail éditorial qu'il importe de dégager et d'analyser à partir de l'observation du livre. Si l'on considère le livre comme le résultat d'un travail sur le texte et l'image dans lequel le support formel du livre imprimé, comme le texte luimême, constituent une même séquence spatio-temporelle, éditer consiste en effet à proposer une lecture d'une œuvre écrite ou visuelle par le biais d'un arrangement conceptuel et formel (papier, format, caractères, mise en page mais aussi établisse-

25. Charles Baudelaire, Correspondance, Tome I, II, Édition de Claude Pichois avec la collaboration de Jean Ziégler, Bibliothèque de La Pléiade, Gallimard, 1973.

26. Roxane Jubert, Steven Heller, Margo Rouard-Snowman, Massin et le livre. La typographie en jeu, Massin and books, Typography at play, Archibooks, 2007. S'agissant de l'édition et de la typographie par Massin de La Cantatrice Chauve d'Eugène Ionesco (Gallimard, 1964), le Blog Design et Typo (LeMonde.fr du 2 mars 2006) remarque: "La typographie en tant que medium de transcription phonétique va lui permettre de représenter la scène et les acteurs dans toute leur spatialité. Les échanges, les dialogues prendront corps dans la taille, l'inclinaison, l'anamorphose, le rythme, les graisses, les contrastes, les blancs bien sûr... pour le silence. »

27. Pierre Faucheux, Le Magicien du livre, propos, textes et témoignages recueillis par Marie-Christine Marquat, préface de François Caradec, Paris, Ed. du Cercle de la librairie, 1995.

28. Régis Debray, Introduction à la médiologie, op. cit., p. 146.

29. Ibid., p. 126.

30. Ibid., p. 125.

31. Ibid.

32. Ibid., pp. 137, 141, 144.

33. Pour un développement et des exemples sur cette question, voir B. Ouvry-Vial, «L'éditeur de littérature et la question de la valeur du texte ", in Qu'est-ce qui fait la valeur du texte littéraire?, colloque organisé par Vincent Jouve, 11-12 novembre 2007, URCA. 
ment du texte, présentation, traduction...) qui conditionne le sens et l'interprétation de l'écrit. Plus largement, les techniques matérielles et intellectuelles de transmission témoignent ou sous-tendent une politique éditoriale donnée et choisie - qu'il s'agisse de défendre des savoirs et patrimoines culturels, d'entrer dans la course aux prix littéraires, de publier des romans de gare ou autres. Quelle que soit aussi la valeur éducative, culturelle, sociale ou littéraire d'une politique éditoriale, l'éditeur est un interprète des idées de son temps : il joue le rôle du lecteur, extrapole à partir de sa propre réaction de lecteur, anticipe sur ce que sera celle du public. Il joue aussi le rôle de l'auteur, se met à sa place, s'imprègne de ses intentions. Le livre est le support et l'instrument de communication qui met en relation auteur et lecteur et organise les conditions de leur rencontre.

D’où l'intérêt de considérer les situations de médiation éditoriale sous l'angle de la réception, au double sens de réception du texte par l'éditeur et de réception des marques d'une écriture éditoriale par le lecteur. La première acception du terme vise à établir une phénoménologie de la lecture éditoriale, à en déterminer les critères d'évaluation, à en analyser les traces et les effets possibles dans l'édition du texte et dans l'objet - livre. La seconde pourrait conduire à définir une sensibilité intellectuelle partagée, à montrer comment ce sentiment est mis en œuvre dans les catalogues de maisons d'édition et comment il est reçu par le public. L'une et l'autre acception permettraient de préciser la notion d'interprétation éditoriale à travers diverses pratiques observables, de repérer des constantes entre les conceptions du livre, de l'écriture, de la lecture de différents éditeurs et d'évaluer les conséquences de l'interprétation éditoriale sur la production éditoriale et sa réception.

La réception éditoriale peut être définie a minima comme une situation de lecture productrice d'une forme textuelle évoluée par rapport à la forme originelle, ne serait-ce que par le traitement technique d'impression et reproduction en nombre, que le texte a en passant à la forme livre. Cette situation de lecture productive repose sur une triple démarche de compréhension, interprétation et application du texte $e^{34}$ et même si les critères de référence de cette triple démarche et ses enjeux sont à la fois complexes et spécifiques, on peut d'emblée la qualifier d'herméneutique, ce qui est moins une manière de promouvoir cet acte de réception, de l'élever au statut supposé supérieur de discipline de la pensée - car il reste avant tout une pratique - que d'apparenter cette pratique à une science du texte dès lors accessible à l'observation phénoménologique et susceptible d'être formalisée.

Il ressort de cette définition que le travail éditorial - lecture et mise en livre est un acte de réception spécifique distinct de la réception critique et de la réception publique, celle du lecteur "ordinaire" même s'il entretient avec l'une et l'autre des liens qu'il nous appartient aussi de préciser. Elle est proche de la critique littéraire par la démarche herméneutique, éventuellement par les modèles esthétiques auxquels elle se réfère, par son enjeu de médiation ou de

34. Triade définie par H.R. Jauss, Pour une esthétique de la réception, Gallimard, 1972 et reprise dans Pour une herméneutique littéraire, traduit de l'allemand par Maurice Jacob, Bibliothèque des Idées, Gallimard, 1988, p. 17. Pour leur application à la lecture éditoriale, cf. Brigitte Ouvry-Vial, «Le Savoir-lire de l'éditeur ? Présupposés et modalités ", in Figures de l'éditeur, dir. par Bertrand Legendre et Christian Robin, Nouveau Monde édition, 2005, pp. 227-244. 
transmission de la littérature sur la base d'un jugement approfondi ${ }^{35}$, mais elle s'en distingue radicalement en ce qu'elle n'aboutit pas à un discours second, à un autre texte. La sentence ou caution exprimée est tout entière contenue dans l'existence même du livre imprimé et la trace de la lecture ou de l'interprétation éditoriale sera tout au plus repérable dans un ensemble de signes discrets que le lecteur identifie difficilement comme un jugement soit parce qu'on les confond avec le texte même tel que voulu et écrit par l'auteur - passage à la ligne, paragraphes, illustrations, notes... - soit parce qu'il s'agit de signes apparemment conventionnels et techniques auxquels on accorde une faible valeur critique - interlignage, caractère, format, foliotage -, soit encore, pour le paratexte rédactionnel quatrième de couverture, avertissements, appareil critique, préface -, parce qu'on ne pourrait vraiment en mesurer l'impact critique que par comparaison avec d'autres éditions d'un même texte, ce qui n'est pas toujours possible.

Il y également parenté et distance entre réception éditoriale et réception publique du lecteur ordinaire. Parenté parce que l'éditeur est un lecteur au même titre que d'autre, et même un double du lecteur ordinaire du lecteur cible à qui est destiné l'ouvrage et dont l'éditeur connaît les compétences, envisage la réaction, anticipe le plaisir ou déplaisir. Mais distance en ce que le rôle de l'éditeur est aussi de s'extraire du contexte de réception de son temps ou de son groupe pour proposer à la lecture des textes qui dépassent et forcent l'horizon d'attente supposé du lecteur. Distance aussi en ce que l'éditeur est un double aussi de l'auteur, que la lecture qu'il fait est suivie d'aménagements textuels qui vont préparer, guider, orienter la lecture finale. C'est donc un lecteur omniscient comme en termes de récit diégétique on parle d'auteur omniscient, doté d'un pouvoir et d'un vouloir, même si ses intentions, parce qu'elles accompagnent l'écriture et soulignent les intentions d'auteur telles que l'éditeur les a perçues et comprises, s'effacent derrière ces intentions d'auteur elles et ne sont pas ellesmêmes directement perçues et comprises ou reconnues par le lecteur comme étant celles de l'éditeur. Éditer c'est contribuer à un livre qu'on ne signe pas, c'est, comme le suggère Paulhan à propos de la critique idéale, écrire sans laisser de traces.

Ceci nous amène naturellement à envisager la problématique de la réception éditoriale sous un double aspect: d'un côté, le terme s'entend comme la réception du texte par l'éditeur, la manière et l'esthétique selon laquelle il lit, comprend, interprète et restitue le texte et la lecture qu'il en a faite dans le livre; de l'autre il s'entend comme la réception de la lecture éditoriale par le lecteur qui comprend et mesure et apprécie diversement les modalités et enjeux du travail d'édition du texte ${ }^{36}$. Sachant qu'entre les deux acceptions du terme, la distinction

35. Sur la nécessité et la nature du jugement, cf. Brigitte Ouvry-Vial, «Jean Paulhan et Gaëtan Picon, des éditeurs entre tradition et transmission", in L'acte éditorial. Publier à la Renaissance et aujourd'hui., dir. par B. Ouvry-Vial et A. Réach Ngo, Textuels (à paraître, 2008).

36. Les chercheurs qui travaillent sur la sémiotique de la matérialité du livre et le rôle des imprimeurs libraires $\mathrm{du} \mathrm{XVI}{ }^{\mathrm{e}}$ siècle dans la structuration des narrations, adoptent particulièrement cette deuxième approche. Plus généralement, c'est le cas des travaux d'historiens de la lecture, comme Roger Chartier dont Dorothea Kraus remarque la délimitation par rapport à l'esthétique de la réception: «L'auteur, le texte, le livre et les lecteurs sont les quatre pôles entre lesquels se déploie le champ de travail de

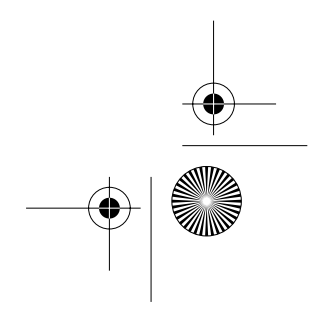


est artificielle - nécessaire seulement à la présentation théorique qu'on en fait puisque la manière dont le lecteur recevra la lecture éditoriale est réputée implicite, pensée et organisée par l'éditeur dans son édition.

\section{CONTRIBUTION DES THÉORIES DE LA RÉCEPTION À L'ÉTUDE DE L'ACTE ÉDITORIAL}

Pour l'étude de la réception éditoriale, entendue comme la réception par l'éditeur du texte d'un auteur, processus mixte de lecture et de mise en livre ${ }^{37}$, la contribution des théories de la réception est d'importance et d'ordres divers. D'abord, contribution par défaut, les théories de la réception sont susceptibles de prendre en charge cette question alors même qu'elle est absente de l'histoire du livre et de la lecture ${ }^{38}$. Ensuite, la réflexion de H.R. Jauss sur l'herméneutique littéraire sert de cadre méthodologique à une approche de l'acte éditorial de la même manière que Jauss a affirmé la pertinence d'une approche de l'herméneutique littéraire à partir de l'herméneutique philosophique de Gadamer : D'un côté pour que l'acte éditorial soit considéré comme acte herméneutique en soi, il faut qu'on puisse y reconnaître l'importance de l'unité des trois moments - compréhension, interprétation, application -, d'un autre côté Jauss admet que l'herméneutique littéraire « a réduit sa théorie à l'interprétation, [elle] n'a jamais articulé son besoin de compréhension et a [tellement] négligé le problème de l'application [...]..$^{39}$ »

Si on suit donc, en l'appliquant non au cas général de l'herméneutique littéraire mais au cas particulier de l'acte éditorial, le protocole ou la préoccupation de Jauss qui est de "saisir la démarche primaire de la compréhension à partir de l'objet esthétique » on est conduit de manière rigoureuse à un certain nombre de questions voire de justifications fructueuses selon lesquelles : la compréhension n'est pas

$\rightarrow$ Roger Chartier sur une histoire du livre et de la lecture et qu'il cherche à relier dans le cadre d'une histoire culturelle du social. Le concept d'"appropriation" rend dans cette perspective non seulement possible de faire converger ces objets de recherche, mais aussi de les mettre en relation avec les pratiques de la lecture qui déterminent chaque appropriation et qui dépendent à leur tour des compétences de lecture d'une communauté de lecteurs, des stratégies de l'auteur, de la forme du texte. Quatre aspects sont essentiels pour chaque forme de réception : premièrement, la compréhension des déterminations par lesquelles la lecture pleine d'inventions des lecteurs est guidée et limitée; deuxièmement, les effets de signification prédisposés par les textes (et donc par l'auteur) eux-mêmes; troisièmement, les restrictions et les influences subies par le lecteur à travers les formes de la transmission du texte ; quatrièmement, les capacités d'une communauté de lecteurs ou bien les conventions de la lecture individuelle. [...] La plus grande importance revient alors à la matérialité du signe : elle révèle tout autant les stratégies de l'auteur et partant la construction d'un lecteur implicite ou visé que les pratiques de la lecture qui s'inscrivent en elle. » ("Appropriation et pratiques de la lecture », Labyrinthe, Thèmes, 13-25 [En ligne], mis en ligne le 7 février 2005, http://revuelabyrinthe.org/ document56.html).

37. Nous y avons consacré quelques travaux auxquels nous nous permettons de renvoyer, notamment B. Ouvry-Vial, «Performance du texte dans le livre ou rôle de l'éditeur dans son accomplissement », op. cit., qui traite des conditions dans et pour lesquelles l'éditeur, au sens performatif du terme, exécute le texte par essence inachevé et en assure l'accomplissement dans le livre, ou « Le savoir lire de l'éditeur? Présupposés et modalités ", in Figures de l'éditeur, op. cit., qui est une description de la méthode spécifique de lecture de l'éditeur et une réflexion sur la compétence éventuelle qu'elle requiert : s'agit-il vraiment d'un savoir ou plutôt d'une praxis?

38. Le constat de cette absence est détaillé dans notre article "Le savoir lire de l'éditeur ? Présupposés et modalités ", op. cit., pp. 228-230.

39. H.R. Jauss, Pour une herméneutique littéraire, op. cit., p. 15.
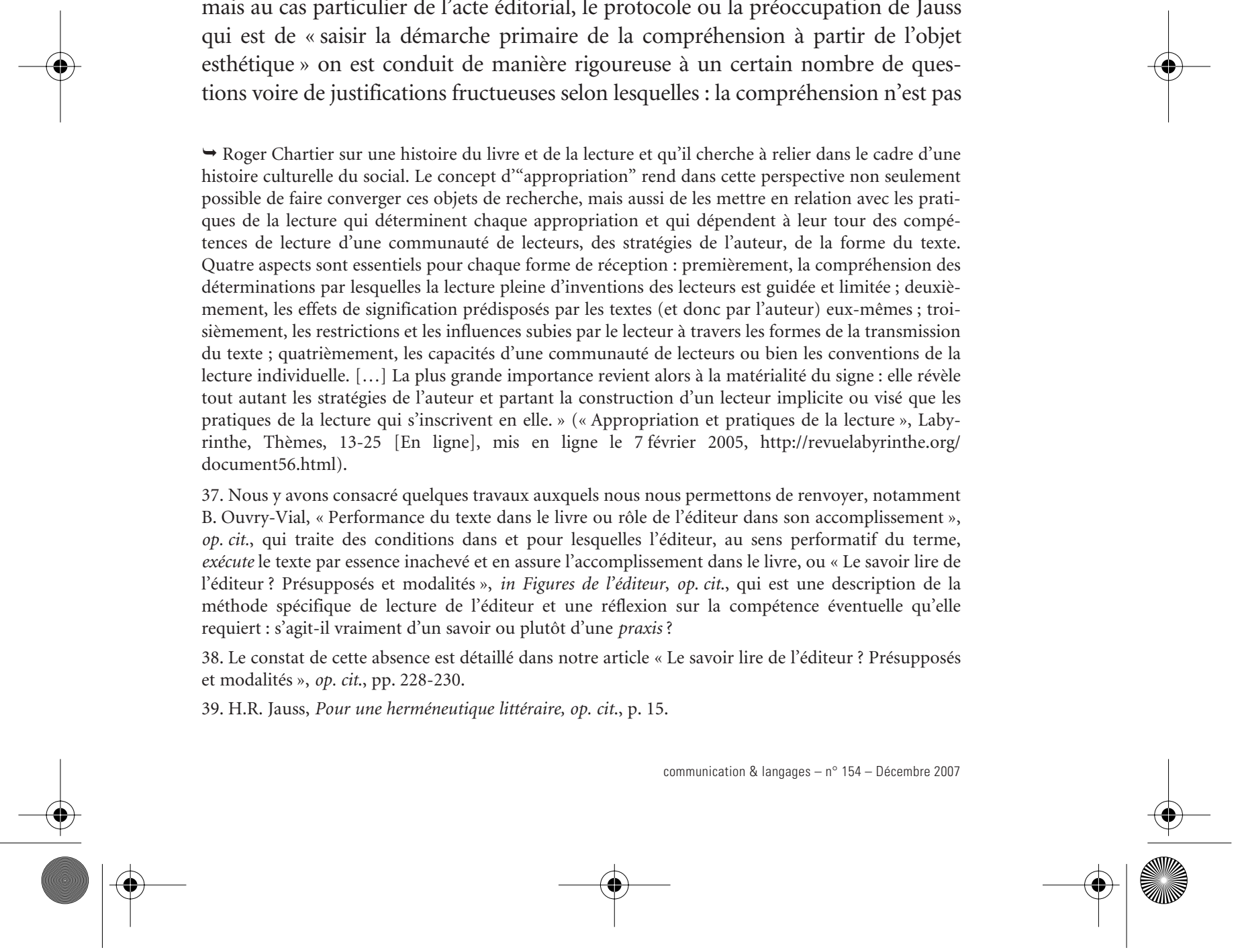
un acte purement contemplatif dans lequel l'interprète efface sa subjectivité ; il est orienté par une visée préalable de l'interrogation, un intérêt de celui qui interroge, intérêt nous dit Jauss en citant lui-même Bultmann ${ }^{40}$ qui «vit également d'une façon ou d'une autre dans le texte [qui est] à interpréter et [qu'il] fonde la communication entre le texte et son interprète ». Plus généralement, la réflexion de Jauss sur l'herméneutique de la question et de la réponse ${ }^{41}$ est instructive pour souligner l'importance de l'esthétique dans l'acte de réception qui donne préséance à la question de l'interprète, grâce à laquelle la réponse du texte est toujours nouvelle. Elle suggère l'idée d'une compréhension moins interprétative ou argumentative qu'interrogative laissant à l'émerveillement, l'étonnement, l'admiration, formes de la réception esthétique qui reviennent explicitement et massivement dans les documents ou témoignages sur l'histoire éditoriale passée ou présente.

Troisième contribution, les théories de la réception formalisent, comme une série de traits repérables et reproductibles, un processus qui parce qu'il n'est observable que dans la pratique semble empirique ou purement intuitif; cette faculté d'étonnement en est un. Un des traits communs aux pratiques éditoriales individuelles (dans le domaine littéraire au moins) serait que la caractérisation esthétique du texte n'est pas une considération consécutive à son interprétation mais au contraire un prémisse, l'amorce d'une interprétation, que l'interprétation est une forme implicite de la compréhension et que pour paraphraser Gadamer cité par Jauss ${ }^{42}$, l'acte éditorial comme processus de compréhension réalise quelque chose qui ressemble à une application du texte à la situation présente de l'interprète. Quel est cet interprète de référence? Le lecteur dont l'éditeur serait un double éclairé, aventureux ? L'éditeur limite-t-il alors sa réception à celle dont le lecteur est supposé capable ou bien suppose-t-il un lecteur le plus doué possible, ou encore adapté au produit éditorial envisagé. L'interprète de référence est-il l'éditeur lui-même en tant qu'intermédiaire entre auteur et lecteur, en position de lecture écrivante? Quelles limites fixe-t-il alors à son intervention? Se coule-t-il dans le moule de l'auteur pour le prolonger et le promouvoir? Comment évite-t-il de se substituer à lui ? La question reste ouverte.

Enfin, l'antériorité et l'acuité particulière du questionnement de Jauss sur l'autonomie possible d'une herméneutique littéraire constitue un modèle d'interrogation sur l'autonomie éventuelle de la réception éditoriale comme herméneutique par rapport aux objets qu'elle se donne; sur son éventuelle spécificité sui generis par rapport à des démarches herméneutiques dépendantes des corpus ou champs disciplinaires qu'elles se donnent; sur la manière donc dont la réception éditoriale «procéderait pour rendre justice au caractère esthétique des textes auxquels elle s'intéresse ${ }^{43}$ sans reproduire ou se cantonner aux critères établis

40. R. Bultman (1961) cité par H.R. Jauss, op. cit., p. 55.

41. Pour une herméneutique littéraire, Ire partie, "L'herméneutique de la question et de la réponse », op. cit., pp. 33-351.

42. «L'idée que [...] l'interprétation est donc la forme explicite de la compréhension, tout comme l'idée que "processus de la compréhension réalise toujours quelque chose qui ressemble à une application du texte à la situation présente de l'interprète" [...] », H.G. Gadamer, Vérité et méthode, trad. franç. de E. Sacre, Paris, 1976, in H.R. Jauss, Pour une herméneutique littéraire, op. cit., p. 14.

43. Ibid., p.12. 
par la critique, la doxa, l'horizon d'attente supposé du lecteur, mais en découvrant, innovant, anticipant plus simplement, tout en étant capable de conduire et guider auteurs et lecteurs à la rencontre les uns des autres.

\section{ÉCRITURE, ÉNONCIATION, GESTE ÉDITORIAL(E) ?}

Les questions que les théories de la réception incitent à poser permettent ainsi à la réflexion sur l'acte éditorial de franchir un cap, de sortir du champ restreint et exclusif de la description historique et technique ou de l'histoire littéraire, d'être institutionnalisée comme un domaine de recherche abordant des corpus de documents imprimés de langues et d'époques variées, à travers l'angle large et complexe des acteurs, des stratégies, des productions, des enjeux sociaux. Le terme d'éditologie, qui pourrait désigner le champ disciplinaire et la méthode fédérant les différents travaux a été élargi par J.-C. Baudet ${ }^{44}$ au sens d'approche sociolinguistique de la science. D'autres approches prennent le terme au sens d'étude des processus sociaux culturels et des procédés matériels et intellectuels qui sous-tendent l'acte éditorial - lecture et mise en livre -, et l'appliquent à un champ éditorial restreint, par exemple les textes de littérature et sciences humaines. On pourrait, sauf à redouter les néologismes, distinguer la démarche éditoriale des études sur l'acte éditorial en employant pour ces dernières le terme d'éditophilologie qui regrouperait autour de l'objet, les directions théoriques et les outils de méthodes développées dans plusieurs champs disciplinaires ${ }^{45}$. L'objet lui-même en effet, induit des convergences et s'il est un constat et principe sur lequel les différentes approches s'accordent c'est la prégnance du support ${ }^{46} \mathrm{dont}$ Régis Debray traduit l'impact épistémologique : «Les supports du goût redéfinissent les œuvres... et les façons d'interpréter [...] il naît de là un changement de perspective qui peut se traduire, en matière littéraire, par un transport d'accent sur l'énonciation plutôt que sur les énoncés. [...] Le modus operandi semblera plus révélateur que l'opus, mais le deuxième, en fait, ne prend effet que par le premier. ${ }^{47}$ »

Il en découle naturellement qu'une des orientations de recherche déjà à l'œuvre consiste à considérer l'acte éditorial comme une situation d'énonciation, non pas dissociée mais intégrée au texte et qui se traduit autant dans et par la matérialité visuelle du livre que dans les opérations intellectuelles de son établissement et

44. Cf. Communication et cognition, vol. 23, 1990.

45. Sociologie, Linguistique, Études Littéraires, SIC, Bibliothéconomie. Par exemple, le groupe GEST (fondé par Brigitte Ouvry-Vial et Anne Réach Ngo en 2004, dans le Cadre de l'École doctorale Langues, Lettres, Image et Civilisation de Paris 7), s'intéresse aux significations des pratiques intellectuelles de médiation au regard desquelles l'histoire culturelle du livre ou de la littérature n'est pas une approche disciplinaire imposant ses règles (historiques ou littéraires) propres mais seulement un terrain d'exploration soumis à des méthodes d'investigation et d'analyse qui lui sont extérieures. Il s'agit délibérément d'affranchir le « livre » du double domaine des études littéraires - où il est encore cantonné en France du fait de la provenance disciplinaire des chercheurs - et des approches historicistes qui s'en sont emparé de longue date comme en témoigne le domaine du Book History, plus développé dans les pays anglo-saxons qu'en Europe.

46. C'est une des pierres de touche liminales des travaux de Roger Chartier, Cf. L'ordre des livres, lecteurs, auteurs, bibliothèques en Europe entre XIV et XVIII siècle, Aix-en-Provence, Alinéa, 1992.

47. Régis Debray, Introduction à la médiologie, op. cit., p. 108.
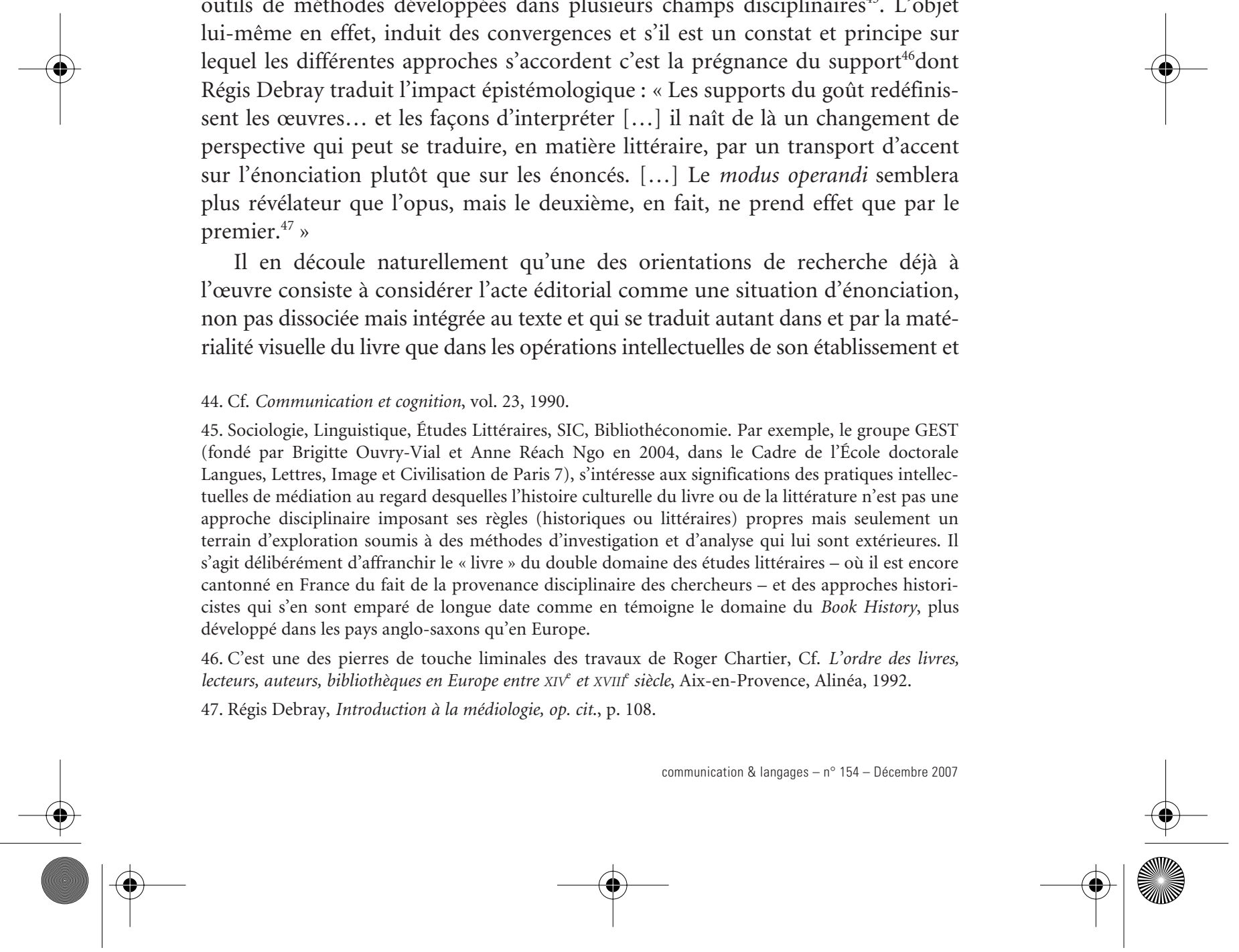
les éléments paratextuels qui l'accompagnent ${ }^{48}$. Si la fonction éditoriale, terme le plus souvent employé, est envisagée dans son évolution au cours des siècles, à travers des figures et dans des situations de production livresque variées, l'ensemble des savoirs sur lesquelles cette fonction repose et des mises en œuvre pratiques auxquelles elle conduit paraissent mieux recouverts par le terme générique d'acte éditorial que nous avons employé jusque-là et qui désigne le processus de transsubstantiation symbolique ${ }^{49}$ du texte en livre dont l'éditeur est responsable. Toutefois, selon l'angle choisi pour interroger ce processus, et en particulier quand on cherche à en identifier et en décrire les traces dans le ou les livres qui en résultent concrètement, le terme d'acte parait lui-même trop général. Aussi, des approches spécifiques proposent-elles des terminologies plus précises pour désigner les différents exercices ou modalités selon lesquelles l'instance éditoriale s'exerce et se donne à voir dans le livre. On retiendra trois de ces catégories, l'écriture éditoriale, l'énonciation éditoriale, le geste éditorial, pour en présenter brièvement, de manière comparative, les enjeux. Écriture éditoriale, énonciation éditoriale, geste éditorial, ne sont en effet pas des termes interchangeables mais complémentaires. Ils désignent trois approches différentes d'un même objet, le livre, qui résulte d'un acte auctorial et éditorial ou d'un acte auctorial partagé entre auteur et éditeur; trois manières différentes aussi dont cet acte matériel et intellectuel s'inscrit dans le livre.

Écriture éditoriale ${ }^{50}$ désignerait, à travers la typographie, la mise en chapitres, l'organisation formelle de l'ouvrage, les traces visibles et lisibles de l'auctorialité éditoriale que l'imprimeur inscrit dans la matérialisation de l'écrit au $\mathrm{XVI}^{\mathrm{e}}$ au moment où il invente le livre. La notion n'est pas nécessairement cantonnée à cette période et on peut en étudier le prolongement ultérieur mais la standardisation des procédés d'impression, des conventions d'édition, l'importance croissante des stratégies commerciales qui prennent le pas dans la politique éditoriale d'ensemble sur la considération du texte en soi, entraînent une dilution et une réduction des traces comme de la marge de manœuvre même de cette écriture. La notion met en effet l'accent sur le «discours du livre» résultant de la ou des pratiques éditoriales considérées et il est certain que le partage, entre auteur et éditeur, de l’autorité productrice du livre, lequel a évolué considérablement au cours de siècles et connu des formes variables, reste une constante. Pour autant, une sémiotique comparée de l'écriture éditoriale à différentes époques de l'histoire du livre ${ }^{51}$ pourrait aboutir au constat d'une quasi-absence ou d'une

48. Emmanuël Souchier remarque fort justement que la conception de Genette du paratexte fait en quelque sorte l'impasse de cette matérialité ou n'en fait pas suffisamment l'expression d'une énonciation éditoriale. Cf. Lire \& Écrire: Éditer des manuscrits aux écrans autour de l'ouvre de Raymond Queneau, Habilitation à diriger des recherches, Université Paris 7-Denis Diderot, 1998, pp. 205-206.

49. L'expression est de Pascal Durand et Anthony Glinoer, in Naissance de l'éditeur, l'édition à l'âge romantique, Les Impressions Nouvelles, Paris-Bruxelles, 2005, p. 182.

50. L'article d'Anne Réach Ngô, dans ce numéro, en propose une synthèse. On peut aussi consulter la thèse d'A. Réach Ngo, "La mise en livre des narrations de la Renaissance : écriture éditoriale et herméneutique de l'imprimé », Paris IV-Sorbonne, décembre 2005.

51. Abordée partiellement dans l'ouvrage en préparation L'acte éditorial. Publier à la Renaissance et aujourd'hui., dir. par B. Ouvry-Vial et A. Réach Ngô, Textuels, UFR LAC/Université Paris 7-Denis Diderot, janvier 2008. 
non-signifiance de l'écriture éditoriale contemporaine par opposition à celle des situations antérieures observables.

Énonciation éditoriale ${ }^{52}$ (qui ne concerne pas simplement la situation du livre et du texte, mais aussi de l'écran) s'attache davantage - dans le domaine de l'ouvrage traditionnel sur papier - à la position de l'editor, chargé de l'établissement d'un texte, editor expert dans le domaine de l'auteur sans être l'auteur mais qui peut être chargé par l'auteur et ou l'éditeur de la conception et constitution du volume. Tenu d'identifier et de respecter les normes et contraintes qui viennent de l'œuvre et celles qui viennent du livre, volume ou collection. Cette double cohérence parfois contradictoire l'amène à choisir et opter radicalement ou alternativement pour une ou l'autre situation d'énonciation. Mais plus spécifiquement, l'apport ${ }^{53}$ de la notion d'énonciation éditoriale, telle que l'a conceptualisée Emmanuël Souchier, c'est de mettre l'accent sur la réalité polyphonique, plurielle du texte mis en livre. On peut objecter que le terme lui-même, plus que la situation d'énonciation à laquelle il renvoie effectivement, a une connotation fortement linguistique qui pourrait sembler, paradoxalement, écarter la matérialité physique de l'objet livre également prise en charge. Toutefois, le concept permet non seulement d'enrichir l'analyse littéraire du texte mais aussi de relire, à la lumière d'enjeux communicationnels jusque-là occultés, l'histoire éditoriale de ce livre à plusieurs voix et plusieurs mains, qui résulte d'un faisceau énonciatif, d'une énonciation démultipliée ${ }^{54}$.

Geste éditorial, un terme qui reste à conceptualiser ${ }^{55}$, concerne davantage la position de l'éditeur médiateur entre auteur et lecteur, assurant la transmission de l'œuvre sur la base d'une lecture, d'une évaluation de la valeur à la fois intellectuelle et économique. Le terme de geste désigne donc dans un premier temps le double acte de lecture et de mise en livre et par extension l'organisation dans le livre des conditions de réception de l'œuvre. Il suppose une interprétation des intentions de l'auteur et de l'horizon d'attente de l'ouvre en même temps qu'une évaluation des compétences et pratiques de lecture des lecteurs. La mise en relation des deux ordres décide des modalités d'édition qu'il s'agisse d'un livre ordinaire, répondant à des normes conventionnelles ou d'un ouvrage à diffusion restreinte exhibant la conscience d'une spécificité du support livre et de son traitement nécessaire.

Les marques visibles et lisibles de ce geste sont avant tout le choix de l'œuvre, genre, auteur, lequel repose sur une mesure de sa valeur et des critères esthétiques. Les choix techniques, format, papier, mise en page, maquette intérieure,

52. Cf. les travaux d'Emmanuël Souchier.

53. «Le concept d'énonciation éditoriale que nous avons évoqué à plusieurs reprises renvoie à l'élaboration plurielle de l'objet textuel. Il annonce une théorie de l'énonciation polyphonique du texte produite ou proférée par toute instance susceptible d'intervenir dans la conception, la réalisation ou la production du livre. » (Lire \& Écrire : Éditer, op. cit., p. 172).

54. On peut suivre l'application de ce concept par exemple dans l'article de Trung Tran, «D'une intention d'éditeur à une énonciation éditoriale : réflexions autour du texte illustré au XVI siècle ", in L'Acte éditorial. Publier à la Renaissance et aujourd'hui, Actes du colloque réunis par Brigitte OuvryVial et Anne Réach Ngô, Textuels, Revue de l'UFR LAC/Université Paris 7-Denis Diderot, janvier 2008.

55. Le terme a été posé à la constitution du groupe GEST. 
enrichissements du texte viennent soutenir et souligner ce choix esthétique, tandis que les commentaires ou indices des motivations de ce choix livrés dans le paratexte, postface ou préface, quatrième de couverture, introduction, notes..., l'explicitent. Bien entendu, c'est la cohérence de l'ensemble de ces marques qui est recherchée et qui traduit une conception éditoriale globale. Les marques sont parfois tangibles et parfois intangibles, l'absence d'indices de l'intervention éditoriale constituant aussi un signe en soi. En effet, le geste éditorial qui suppose un ajustement de l'édition en cours, du livre en cours, à et en fonction d'un projet esthétique plus large, consiste en une mise en situation - en poche, en collection érudite, en Beaux-Livres..., d'écrits ou d'œuvres identifiés en termes de situations de communications. Mais ces situations pour être formalisables ne relèvent pas toutes d'un modèle unique et l'ajustement nécessaire est plus ou moins délicat et complexe $e^{56}$.

En effet, si écriture et énonciation renvoient à une dimension essentiellement discursive de l'acte éditorial comme mode d'expression productif d'une représentation $^{57}$, le geste permet à une sémiotique de l'acte éditorial d'explorer, au-delà du processus linguistique et discursif, de son effet, de l'image et de l'objet visuel résultant, l'action elle-même. Sans entrer dans une étude ou application détaillée de la notion de geste, on peut ici, à partir des réflexions de Julia Kristeva, percevoir le champ des possibles ainsi ouvert :

«La gestualité [...] est susceptible d'être étudiée comme une activité dans le sens d'une dépense, d'une productivité antérieure au produit, donc antérieure à la représentation comme phénomène de signification dans le processus communicatif ; il est donc possible $d$ e ne pas étudier la gestualité comme une représentation $[\ldots]$ mais comme une activité antérieure au message représenté et représentable. [...] Plus que ce message déjà là [le geste] est (et il rend concevable) l'élaboration du message, le travail qui précède la constitution du signe (du sens) dans la communication..$^{58}$ "

De la même manière que l'étude de cette gestualité nécessite pour Julia Kristeva « une étroite collaboration d'anthropologues, historiens de la culture, philosophes, écrivains, et sémioticiens pour cette "sortie de la parole" " ${ }^{59}$, l'étude de l'acte éditorial comme geste implique de " traverser les structures code-messagecommunication ${ }^{60}$, d'introduire la fonction édition qui est restée jusque-là impensée dans l'approche littéraire du livre, donc de renverser la réflexion sur le système sémiotique du livre en revenant à cet "espace infraordinaire ${ }^{61}$ où il s'élabore. Cette fonction, que nous désignions plus haut sous le terme d'intention

56. C'est le cas par exemple de la situation de communication à la fois type et spécifique qu'est la littérature contemporaine et étrangère, qui, tout en étant "en situation ", ne relève pas d'un modèle unique de situation.

57. Cf. Emmanuël Souchier : «Le paratexte est le mode d'expression privilégié de l'énonciation éditoriale », Lire \& Écrire: Éditer, op. cit., p. 206.

58. Julia Kristeva, $\sum \eta \mu \varepsilon \omega \tau ๘ \eta$, Recherches pour une sémanalyse, chap. 2, «Le geste, pratique ou communication? », Le Seuil, coll. « Points», 1969, p. 32.

59. Ibid., p. 33.

60. Ibid., p. 32.

61. Emmanuël Souchier, op. cit., p. 206.

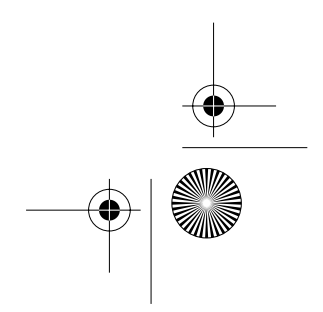


ou intentionnalité, nous pouvons désormais l'élargir et en emprunter le terme à l'étude des systèmes sémiotiques primitifs, des principes d'écriture, qui signalent l'indication ou indication d'action comme principe complémentaire de la représentation $^{62}$; et qui soulignent «l'antériorité synchronique [...] non pas d'un concept par rapport à une voix (signifié-signifiant) mais d'un geste de démonstration, de désignation [...] pour englober dans un même texte sémiotique ${ }^{63}$, le "sujet", "l'objet" et la "pratique". ${ }^{64}$ "C'est à l'analyse de cette fonction - désignation, indication d'action - que nos recherches sur le geste éditorial devraient désormais se consacrer.

De ce point de vue, le geste engloberait écriture et énonciation. Il suppose l'énonciation et engage l'écriture éditoriale, mais ne s'y réduit pas. Il ne correspondrait pas non plus aux mêmes époques de conception de la fonction éditoriale: Écriture éditoriale considérerait une époque où le livre s'invente concrètement dans l'atelier de l'imprimeur ; énonciation une époque où l'éclatement de supports valorise la position de l'editor - auctor et lector à la fois - ; geste serait une notion plus transhistorique correspondant à des figures ponctuelles repérées au cours de l'histoire de l'édition, généralisées au $\mathrm{XX}^{\mathrm{e}}$ et sans doute moins nombreuses au XXI ${ }^{\mathrm{e}}$. La fonction symbolique de l'éditeur, instaurée à la fin du XIX ${ }^{e}$ par suite du sacre réciproque de l'auteur et de l'éditeur ${ }^{65}$, trouve son plein-emploi au $\mathrm{XX}^{\mathrm{e}}$; mais au $\mathrm{XXI}^{\mathrm{e}}$, la concurrence avec d'autres supports - et surtout d'autres modes et valeurs d'appropriation de la culture et du savoir, la renvoie à une dimension artisanale. Le geste doit être pensé, dans sa pratique et sa finalité, en mettant l'accent, en particulier, sur le principe d'autonomie de la fonction éditoriale et de la personne qui l'exerce, face aux contraintes économiques et sociales de son temps et, face à la question du temps en général, sur sa visée de transmission : non seulement le geste éditorial consiste à «transporter une information dans le temps ${ }^{66}$ mais il «se déroule aussi dans le temps ${ }^{67}$, sous forme de processus et d'après une succession plus ou moins réglée.

On ne peut pour autant pas dire qu'écriture, énonciation, geste, correspondent à trois âges du livre; mais il est certain que pour chacune de ces notions, le contexte de son exercice doit être pris en compte, par comparaison avec ce qui précède et ce qui suit. Il semblerait en effet que écriture, énonciation et geste se rejoignent dans l'intention - de ceux qui s'y sont diversement, chacun à sa manière, appliqués ou illustrés -, d'inscrire leur temps dans les livres et que leur temps, par eux ainsi transmué, fasse époque. Si ces trois notions méritent d'être étudiées de manière approfondie, et appliquées à des cas d'espèces les plus diversifiés possibles, le croisement rapide qu'on vient d'en proposer témoigne - alors même que "l'objet livre a été l'impensé de la théorie littéraire [comme] l'objet

62. J. Kristeva, $\Sigma \eta \mu \varepsilon \omega \tau \lll \kappa \eta$, op. cit., p. 34.

63. C'est-à-dire le livre.

64. Ibid.

65. Constaté par Pascal Durand et Anthony Glinoer, in Naissance de l'éditeur, op. cit., p. 27 et chapitre 3.

66. Régis Debray, Introduction à la médiologie, op. cit., p. 3.

67. Ibid., p. 4.

communication \& langages - n 154 - Décembre 2007 
d'art, dans sa matérialité, a été l'impensé des esthétiques classiques ${ }^{68}-$ qu'il est permis d'envisager les études sur le livre et l'édition de manière transversale et de fonder l'acte éditorial comme un objet théorique d'analyse dans le champ des études de la transmission et réception de l'écrit en général et de la littérature en particulier.

\section{BRIGITTE OUVRY-VIAL}

68. Ibid., p. 65.

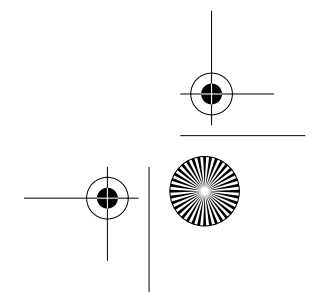

